\title{
CIÊNCIA'NATURA
}

\section{Análise multivariada de dados socieconômicos: um retrato da modernização agropecuária nos COREDEs do Rio Grande do Sul}

Multivariate analysis of socio-economic data: a snapshot of the livestock and agriculture modernization at Rio Grande do Sul's COREDEs

\section{Rafael Pentiado Poerschke', Fernando de Jesus Moreira Junior"}

\begin{abstract}
RESUMO
O processo de modernização da agropecuária brasileira ganhou expressão doravante a organização do sistema financeiro e de crédito rural nos anos 1960. O resultado desse processo no Rio Grande do Sul (RS) se deu de forma heterogênea nas diferentes regiões do estado. A utilização da estatística multivariada permite identificar os diversos níveis de modernização na agropecuária dos Conselhos Regionais de Desenvolvimento (COREDEs) agrícolas em 1995/96. No Rio Grande do Sul, o então estágio de modernização confirma a hipótese inicial, pois os dados evidenciaram uma forte concentração da agropecuária moderna no grupo formado pelos municípios do COREDE Fronteira Oeste. Contudo, um segundo grupo apresentou uma situação moderna, mas não tão voltado para a criação de gado. $O$ grupo 3 demonstrou evidências de transição da agricultura mais tradicional para um padrão diferenciado e caracterizado pela inclusão de insumos modernos. Um último grupo, e de tamanho considerável, foi caracterizado pelo padrão agropecuário mais tradicional.
\end{abstract}

Palavras-chave: Análise de Agrupamentos. Análise Fatorial. Dados Censitários.

\section{ABSTRACT}

Brazilian agriculture modernization process has gained expression henceforth the financial and rural credit system organization in the 1960s. This process result in the Rio Grande do Sul (RS) was heterogeneous in different regions of the state. The use of multivariate statistics makes it possible to identify the different levels of modernization in Regional Agricultural Development Councils' (COREDEs) agriculture in 1995/96. In the Rio Grande do Sul, the then stage of modernization confirms the initial hypothesis since data showed a strong concentration of modern agriculture in the group formed by the cities of the West Border COREDE. However, a second group presented a modern but not so livestockoriented situation. Group 3 showed evidence of a transition from more traditional agriculture to a differentiated pattern featured by the inclusion of modern inputs. One last group, of a substantial size, was featured by the more traditional farming pattern.
\end{abstract}

Keyword: Cluster Analysis. Factor Analysis. Census Data. 


\section{INTRODUÇÃO}

Com importância econômica e histórica, a agropecuária do Rio Grande do Sul (RS) também se beneficiou das políticas verticais do governo federal e estadual voltadas ao setor. Entre 1940 e meados dos anos 1980, o título de celeiro do país, rendeu para o RS uma parcela significativa de recursos do Sistema Nacional de Crédito Rural (FÜRSTENAU, 1988). Nos anos 1990 a matriz do estado pouco mudou, enquanto a fronteira agrícola havia se deslocado para o centro-oeste do Brasil. Dez anos mais tarde a fronteira agrícola já havia alcançado com mais força a região Norte. Mesmo com o deslocamento da fronteira agrícola, o complexo agropecuário do RS não perdeu importância e representava 40\% do total das exportações gaúchas em 2006, e 1,62\% do total exportado pelo Brasil. Na primeira metade dos anos 2000, a agropecuária respondeu, em média, por aproximadamente 9\% do Valor Adicionado Bruto $^{1}$ (VAB) do estado (FEE, 2007).

Na ocasião do desenho desta pesquisa, houve a publicação de um estudo realizado com dados do Censo Agropecuário 1995/96 somados a base de dados da Fundação de Economia e Estatística (FEE). A gestão de Germano Rigotto (2003-2006) buscou medir o desempenho dos Conselhos Regionais de Desenvolvimento (COREDEs) do estado do RS. O estudo chamado de Rumos 2015, mapeou a predominância da atividade econômica nos COREDEs a fim de traçar o perfil de cada Conselho. O estudo revelou um Rio Grande do Sul heterogêneo regionalmente, variando de COREDEs mais agrícolas, enquanto outros possuem padrões que indicam uma indústria pujante. Ainda, alguns COREDEs apresentaram certo equilíbrio entre as atividades na indústria e agropecuária.

No entanto, existia uma visível dicotomia entre os COREDEs em suas especializações, que variaram da monocultura da soja até regiões dinâmicas e bem articuladas na produção de fumo, que é predominantemente familiar. Considerando esse olhar, o presente estudo busca relacionar os municípios que fazem parte dos COREDEs agrícolas segundo seu grau de modernização agropecuária. Para isso,

\footnotetext{
${ }^{1}$ Valor Adicionado Bruto a preço básico é resultado da diferença entre o Valor Bruto da Produção (VBP), a preços do produtor, e o Consumo Intermediário $(\mathrm{CI})$, a preços de mercado. E, o Produto Interno Bruto a preço de mercado é igual ao Valor Adicionado a preço básico, subtraído dos serviços de intermediação financeira indiretamente medidos e adicionado dos impostos sobre produtos, livres de subsídios.
} 
levantamos duas hipóteses fundamentais: em função de uma realidade evidentemente multifacetada encontrada no Rio Grande do Sul, existe uma diferença significativa nos padrões e níveis de modernização da agropecuária entre os COREDEs predominantemente agrícolas do RS; o mesmo espera-se entre os municípios que compõem determinado COREDE.

Na ocasião da redação desse estudo, havia a expectativa dos dados do Censo Agropecuário de 2006 do IBGE. Portanto, o presente trabalho buscou ter um alcance além do problema de pesquisa, o qual propunha medir o grau em que se encontrava a modernização agropecuária nos COREDEs predominantemente agrícolas ${ }^{2}$ com base nos dados censitários coletados em 1995/96 pelo Instituto Brasileiro de Geografia e Estatística (IBGE). Não menos importante, havia uma segunda motivação no trabalho, pois procurava-se estabelecer e apresentar uma rotina clara para analisar dados de Censo para o Brasil. Uma vez trabalhada com os dados pesquisa imediatamente anterior, seria possível fazer um comparativo de dois retratos da modernização agropecuária do estado, além de disponibilizar a metodologia para que outros pesquisadores pudessem fazer o mesmo levantamento em seus estados ou regiões com o censo dos anos 2000.

Assim, o presente estudo teve por objetivo, em virtude do elevado número de variáveis (17) e municípios (139), formar grupos o mais homogêneos possível por meio da análise de agrupamentos e discriminante. Procuramos também identificar os fatores que representam com mais fidelidade a variação dos dados originais através da análise fatorial. Após agrupados e encontrado os fatores, também destacamos os padrões de modernização existente nos grupos formados pelos COREDEs agrícolas gaúchos em 1995/96. O trabalho está dividido em quatro seções, sendo a primeira essa introdução ao problema, seguida da revisão de literatura, uma terceira que versa sobre a metodologia e o referencial teórico. Por fim, uma última seção reúne e discute os principais resultados do estudo proposto.

${ }^{2}$ No ANEXO A encontra-se o mapa do estado do Rio Grande do Sul e os COREDEs em questão. 


\section{REVISÃO DE LITERATURA}

A ideia de um Brasil moderno, pelos militares, marcaria a economia nacional até a instauração do Plano Real. Após os anos 1960, a agricultura brasileira manteve sua importância enquanto assunto de Estado. No referido momento da história, as políticas públicas buscaram além de modernizar, aqui inclui-se o sistema de crédito, diversificar a produção agrícola nacional.

Sabe-se que o período denominado de "milagre econômico" - final da década de 60 - promoveu o crescimento recorde da economia. O Brasil, por sua "vocação agrícola", teve a agricultura beneficiada pelo processo de modernização que acontecia no país, favorecida principalmente por uma política de crédito agrícola voltada para custear a safra e financiar aumentos horizontais na área plantada via investimentos em tecnologia e infra-estrutura. No período de 1965-79, instaurou-se a fase de expansão no financiamento agrícola, mediante recursos federais, marcando o auge do Sistema Nacional de Crédito Rural (SNCR). O SNCR ofereceu crédito abundante e taxas de juros convidativas para estimular a aquisição dos insumos considerados pelo governo como modernos e indispensáveis (MASSUQUETTI, 1998).

Nos anos 80, quando configurada uma crise fiscal do Estado, a estratégia de desenvolvimento agrícola baseado no crédito rural subsidiado se encontrava em decadência, alterando o modelo que até então vinha sendo defendido. Com o aperto fiscal, houve um maior controle dos gastos públicos, com isso a tendência dos dispêndios relacionados com a agricultura resultaram na remoção de subsídios e políticas verticais utilizados. O setor agropecuário frente aos desmantelamentos dos instrumentos de intervenção na agricultura, que foram intensamente utilizados desde os anos 60 e 70, buscou novas formas de financiamento, custeio e investimento. 0 crescimento do produto deveria ir além do constante aumento via áreas cultivo ${ }^{3}$ e superar o modelo anterior. A agropecuária precisou se voltar para um crescimento da produção que fosse oriundo de rendimentos físicos, calcados na produtividade e racionalização do uso do solo (GRAZIANO DA SILVA, 1996).

\footnotetext{
${ }^{3}$ Salienta-se que a ênfase das políticas de crédito por parte do governo no período, que foram sempre na medida de procurar aumento no produto agrícola, terminaram em uma expansão via aumento da área utilizada. Interessante é o fato de que, no período, a marca sempre foram os baixos níveis alcançados de produtividade pela agricultura nacional.
} 
Até a década de 50, as culturas mais desenvolvidas, como arroz e trigo plantado desde o século XIX - sofriam com a competitividade externa. Tal situação favoreceu a inserção da sojicultura nos COREDEs agrícolas do estado. Schneider e Fialho (2000, p. 127) apontaram que a frente da expansão da soja estava concentrada na zona colonial do noroeste do estado (COREDEs Noroeste Colonial, Missões e Médio Alto Uruguai), tendo sido sua difusão baseada nos "incrementos anuais de área plantada".

A velocidade do crescimento da produtividade média ultrapassou a incorporação de novas áreas plantadas após os anos 1990. O aumento do volume de produção aponta para uma maior atenção, por parte do produtor rural, em investir em tecnologia para elevar a produtividade, superando o modelo característico que predominou até meados dos anos 70. Partindo da ideia de que o tamanho do solo é uma das muitas atividades que absorvem o tempo e os recursos do agricultor (RANGEL, 2004), então, frente às dificuldades encontradas nos anos 1980, o produtor precisou racionalizar o uso das áreas agrícolas. A mudança de objetivo dos produtores aconteceu, pois produtividade e redução de custos passaram a ser prioridade frente à incorporação de novas áreas para cultivo. Essa etapa de preocupação voltada para o rendimento, é mais efetiva após os anos 90 e marca um novo momento do processo histórico de modernização da agricultura.

As transformações ocorridas no noroeste, fizeram com que o modo de vida colonial fosse substituído pela cultura tecnificada e integrada aos complexos agroindustriais. Na região sul (que compreende os COREDEs Sul e Litoral) e oeste (COREDEs Fronteira Oeste e Missões) do Rio Grande do Sul onde predomina a produção irrigada e a pecuária extensiva, a dinâmica foi diferente. Na década de 80, a produção de arroz irrigado acompanhou o processo de aumento de produtividade, enquanto a pecuária se manteve estagnada (SCHNEIDER; FIALHO, 2000).

A abertura econômica que se iniciou a partir do Plano Collor, marcou o aumento gradual nas perspectivas comerciais no sistema multilateral de comércio. Esse fator trouxe novas opções de insumos e, consequentemente, uma maior competição do setor em relação às pressões externas. Assim, o acirramento da concorrência foi um incentivo para aumentos de produção baseados na 
produtividade. Uma das variáveis mais importantes que auxiliaram no crescimento da produtividade pode ser atribuída à disponibilidade do estoque e ao domínio de tecnologias geradas pela pesquisa e assistência técnica nas décadas de 70 e 80 . Aqui, destaca-se a criação ou modernização das instituições de pesquisa que tinham como objeto a agropecuária no Brasil. O agricultor aumentou a produtividade da lavoura e ajustou-se a uma nova realidade, com maior risco econômico e de rentabilidade duvidosa com paulatino afastamento do Estado. De fato, foram eficientes até os anos 1980, pois garantiram que o período não fosse o de "uma década perdida" para o produtor rural.

Para Bandeira (1999), o espaço na instância político-administrativa que existe entre estado e municípios constitui um obstáculo para a articulação na abrangência micro-regional. Nesse sentido, se estabeleceram no Rio Grande do Sul os COREDEs, como mecanismos sólidos de participação como um esforço prévio de construção institucional e para conscientizar a comunidade sobre a natureza dos problemas regionais, buscando ações voltadas para superação desses problemas.

O presente estudo deve focalizar a análise sob a ótica desses Conselhos Regionais de Desenvolvimento do Rio Grande do Sul a fim de enriquecer o conhecimento sobre a agropecuária no estado, em especial, nas regiões com predominância da atividade agrícola.

\section{METODOLOGIA E FONTE DE DADOS}

A aplicação de técnicas multivariadas - análise de agrupamentos, função discriminante e fatorial - permitiu identificar os padrões de modernização nos municípios gaúchos. Frente ao elevado número de municípios em análise, tornou-se possível hierarquizar os municípios além de identificar as variáveis mais relevantes para inferência sobre os potenciais de desenvolvimento ${ }^{4}$ dos municípios.

Foram avaliados 139 municípios do estado do RS sob a ótica de dezessete variáveis. Os municípios fazem parte dos COREDEs essencialmente agrícolas,

\footnotetext{
${ }^{4}$ Para rodar essa matriz foram utilizados os softwares Statistical Package for Social Sciences ${ }^{4}$ (SPSS) e Statistica 7.0 disponibilizado pelo departamento de Estatística da Universidade Federal de Santa Maria - Centro de Ciências Naturais e Exatas.
} 
conforme classificação da Fundação de Economia e Estatística (FEE) junto ao Governo do estado do Rio Grande do Sul (2005):
i) Fronteira Oeste, com 10 municípios;
ii) Jacuí Centro, com 6 municípios;
iii) Missões, 21 municípios;
iv) Noroeste Colonial, com 29 municípios;
v) Médio Alto Uruguai, também, com 29 municípios;
vi) Nordeste, com 18 municípios;
vii) Alto da Serra do Botucaraí, com 14 municípios e;
viii) Alto Jacuí, com 12 municípios.

As variáveis levantadas sobre os municípios dos referidos COREDEs foram divididas em três dimensões. A primeira delas compreende as variáveis agropecuárias, que são oriundas na totalidade do Censo Agropecuário 1995/96 e medem, stricto sensu, o nível de modernização agropecuária, conforme a literatura consultada (Quadro 1).

Uma segunda dimensão, agora socioeconômica, é representada pela população rural e população economicamente ativa, também do Censo 1995/96 aliadas ao índice do bloco renda ${ }^{5}$ dos municípios calculados pela Fundação de Economia Estatística do estado do Rio Grande do Sul. Considerando que, segundo Gama (2007, p.27), no Rio Grande do Sul, o transporte de cargas e mercadorias está baseado praticamente no modal rodoviário, ou seja, "90\% do total do estado". Sendo assim, optou-se pela inclusão de uma variável espacial, a qual consiste na distância entre o município e o porto de Rio Grande (RS), por onde escoa maior parte da safra de grãos exportada no RS.

\footnotetext{
${ }^{5}$ Este indicador medido pela FEE leva em consideração o Valor Adicionado Bruto (VAB) no comércio, alojamento e alimentação e Geração de Renda per capita. O presente trabalho versa sobre os COREDES essencialmente agrícolas, dessa forma justificando a utilização do bloco renda que, aparentemente em sua medida, mede o todo do município, mas, como em tais COREDES, os reflexos da variação do bloco renda tem forte ligação com o campo - lembrando que são predominantemente agrícolas.
} 
Quadro 01 - Variáveis responsáveis pela modernização da agropecuária nos COREDEs agrícolas no Rio Grande do Sul.

\begin{tabular}{|c|c|c|}
\hline \multicolumn{2}{|c|}{$\begin{array}{l}\text { Variáveis } \\
\text { agropecuárias }\end{array}$} & \multirow{2}{*}{$\begin{array}{l}\text { Descrição } \\
\begin{array}{l}\text { Assistência Técnica Especializada na } \\
\text { estabelecimentos no RS }\end{array}\end{array}$} \\
\hline 01 & ASSPEC & \\
\hline 02 & ASSAGR & $\begin{array}{l}\text { Assistência Técnica Especializada na Produção Vegetal/total de } \\
\text { estabelecimentos no RS }\end{array}$ \\
\hline 03 & TRATOR & Número de tratores/total de tratores no RS \\
\hline 04 & MAQCOL & Números de máquinas para colheita/ total de máquinas para colheita no RS \\
\hline 05 & ADUBOS & $\begin{array}{l}\text { Número de estabelecimentos que utilizam adubos químicos/total de } \\
\text { estabelecimentos no RS }\end{array}$ \\
\hline 06 & IRRIGA & $\begin{array}{l}\text { Número de estabelecimentos que utilizam sistemas de irrigação/total de } \\
\text { estabelecimentos no RS }\end{array}$ \\
\hline 07 & RECVEG & Valor das receitas advindas da venda de produtos vegetais/valor total no RS \\
\hline 08 & RECANI & Valor das receitas advindas da venda de animais/valor total no RS \\
\hline 09 & FINANC & Valor dos financiamentos nos municípios/valor total no RS \\
\hline 10 & VALVEG & Valor da produção vegetal/valor total no RS \\
\hline 11 & VALANI & Valor da produção animal de grande porte/valor total no RS \\
\hline 12 & AREXPL & Área explorada nos municípios/total da área do RS \\
\hline 13 & GADO & Efetivo de animais de grande porte do município/total do RS \\
\hline \multicolumn{3}{|c|}{$\begin{array}{l}\text { Fonte: Todas as variáveis acima são oriundas do Censo Agropecuário do IBGE 1995/96, assim como, a } \\
\text { variável PEA e POP que seguem. }\end{array}$} \\
\hline \multicolumn{2}{|c|}{$\begin{array}{l}\text { Variáveis } \\
\text { Sócioeconômicas }\end{array}$} & Descrição \\
\hline 14 & POP & População rural do município/total da população rural no RS \\
\hline 15 & PEA & $\begin{array}{l}\text { População economicamente ativa do município/total da população } \\
\text { economicamente ativa no RS }\end{array}$ \\
\hline 16 & RENDA & Índice bloco renda no município \\
\hline \multicolumn{3}{|c|}{$\begin{array}{l}\text { A variável RENDA é um índice utilizado no Índice de Desenvolvimento Socioeconômico para o Rio } \\
\text { Grande do Sul mensurado pela FEE. O Idese é um índice sintético, inspirado no IDH, que abrange um } \\
\text { conjunto amplo de indicadores sociais e econômicos classificados em quatro blocos temáticos: } \\
\text { Educação; Renda; Saneamento; Domicílios; e Saúde todos variando entre } 0 \text { e } 1 \text {. }\end{array}$} \\
\hline \multicolumn{2}{|c|}{ Variável Espacial } & Descrição \\
\hline 17 & DISTRG & $\begin{array}{l}\text { Distância do município em relação à cidade portuária de Rio Grande/total das } \\
\text { distâncias em relação ao porto }\end{array}$ \\
\hline
\end{tabular}

\subsection{Análise multivariada de dados}

A análise multivariada é um ramo da análise estatística que verifica a relação entre grupos de variáveis dependentes e coletadas sobre o mesmo indivíduo (MORRISON, 1976). As $n$ observações sobre $m$ variáveis constituem a matriz de dados, 
em que, $n>m$. Assim, a exigência para aplicação é que o número de variáveis seja menor que o de observações.

O método de Análise Multivariada tem como objetivo otimizar a interpretação de grandes conjuntos de dados. Assim, pretende-se adotar o método na análise econômica, em especial, para verificar os níveis de modernização da agropecuária nos COREDEs do Rio Grande do Sul (RS) tendo como fonte de dados cross section do Censo Agropecuário de 1995/1996. A análise multivariada, de regra, está divida em análise de agrupamentos (AA), função discriminante, análise fatorial (AF), das componentes principais (ACP), dentre outros. Estando de acordo com o sentido do método, este trabalho segue ordinalmente a apresentação sumária supra-exposta de cada técnica, a começar, pelo agrupamento dos cases propostos para o trabalho refinando a técnica com a utilização da separação pela função discriminante, passando a análise das componentes principais e análise fatorial.

\subsubsection{Análise de Agrupamentos (AA)}

A análise de agrupamentos é uma maneira para agrupar um grande número de indivíduos em grupos discretos. A tentativa de agrupar indivíduos ou variáveis é um método mais primitivo em relação a classificação, pois ao agrupar não se sabe a priori o número de grupos ou das características da estrutura interna de cada grupo. A AA é realizada com base na similaridade ou dissimilaridade entre as variáveis a serem analisadas (JOHNSON; WICHERN, 1998, p.725-729). Sendo assim, as observações serão agrupadas por apresentarem características similares de acordo com algum critério de classificação - o algoritmo aglomerativo.

Contudo, para a classificação é preciso que se defina um método de aglomeração, assim como a medida utilizada para mensurar a distância entre os grupos formados. É necessário a escolha de um coeficiente que quantifique o quão parecidos dois ou mais indivíduos são. Esse coeficiente diz respeito à estimação de uma medida de similaridade ou dissimilaridade entre indivíduos a serem agrupados no nosso caso, os municípios dos COREDEs agrícolas. Na medida de similaridade, quanto maior o valor observado, mais parecidos serão os indivíduos. Enquanto na dissimilaridade, o contrário se verifica, ou seja, quanto maior o valor observado, 
menos parecidos eles serão. Ainda, o método de escolha poderá seguir uma sequência que seja aglomerativa, partindo de grupos individuais, ou pode ser feito partindo de um único grupo e, ao se concluir o algoritmo, o resultado final serão grupos com único elemento.

A distância euclidiana entre dois pontos no plano é a mais difundida e utilizada na análise de agrupamentos. Contudo, vale lembrar que se recomenda padronizar as variáveis antes de calcular qualquer distância. Para formas de agrupamento Ward e centróide, a distância euclidiana quadrada é a mais recomendada (HÄRDLE; SIMAR, 2003). A distância euclidiana para um conjunto de dados forma uma matriz que tem como elementos a distância entre duas observações em $n$-dimensão, com $\boldsymbol{x}=$ $\left[x_{1}, x_{2}, \ldots, x_{n}\right]^{\prime}$ e $\boldsymbol{y}=\left[y_{1}, y_{2}, \ldots, y_{n}\right]^{\prime}$

$$
\begin{aligned}
& d(\boldsymbol{x}, \boldsymbol{y})=\sqrt{\left(x_{1}-y_{1}\right)^{2}+\left(x_{2}-y_{2}\right)^{2}+\cdots+\left(x_{n}-y_{n}\right)^{2}} \\
& =\sqrt{(\boldsymbol{x}-\boldsymbol{y})^{\prime}(\boldsymbol{x}-\boldsymbol{y})}
\end{aligned}
$$

Contudo, trabalhamos com a distância estatística entre as mesmas duas observações, assim (1) passa a ser escrita como

$$
d(\boldsymbol{x}, \boldsymbol{y})=\sqrt{(\boldsymbol{x}-\boldsymbol{y})^{\prime} \boldsymbol{A}(\boldsymbol{x}-\boldsymbol{y})}
$$

Sendo $\boldsymbol{A}=\boldsymbol{s}^{-1}$, em que $\boldsymbol{S}$ contêm é matriz de variância e covariância da amostra. Contudo, o método de Ward se adapta bem a medida euclidiana quadrada, expressa por

$$
d e q_{n}^{2}=\sum_{p=1}^{n}\left(x_{n}-y_{n}\right)^{2}
$$

Optou-se por seguir um algoritmo na forma aglomerativa e, por esse motivo, essa forma parte de $n$ grupos de apenas um indivíduo cada, que vão sendo agrupados sucessivamente até que se encontre um último grupo que incluirá a totalidade dos $n$ indivíduos ou as $m$ variáveis em questão.

Os procedimentos hierárquicos usam cinco algoritmos aglomerativos diferentes para desenvolver os agrupamentos, o método de ligação individual, ligação completa, ligação média, o método de Ward e o método centróide (JOHNSON; WICHERN, 1998, p.739). Para o presente estudo, o algoritmo que mais se adequou aos 
objetivos foi o de Ward. Assim, o método de Ward ${ }^{6}$ caracteriza-se pela "formação de grupos com alta homogeneidade interna e muito apropriado para variáveis quantitativas" (MINGOTI, 2005, p.178). Esse método resume-se aos seguintes passos: a) são calculadas as médias das variáveis para cada grupo; b) é calculada a distância euclidiana quadrada; c) somam-se as distâncias para todos os indivíduos; e; d) buscase otimizar a variância mínima dentro dos grupos da relação entre os indivíduos e a média do grupo (centróide).

\subsubsection{Função Discriminante}

A função discriminante abrange duas principais funções. Por um lado, seu emprego permite a alocação de indivíduos em grupos previamente definidos. Ainda, se a intenção não é classificar novos indivíduos, essa função serve para refinar a técnica da análise de agrupamentos efetuada previamente. Assim, a utilização do método será adotado no intuito de verificar a real localização dos municípios dentro dos grupos definidos a priori com o método de Ward.

Para esse estudo, optamos pela generalização da Função Discriminante de Fisher, a qual sugere encontrar combinações lineares univariadas que maximizam a diferença entre os grupos. Para $p$ grupos serão encontradas $p-1$ funções de Fisher. Em virtude da complexidade do cálculo matricial necessário para a estimação das funções, sugere-se ver Johnson e Wichern (1992).

\subsubsection{Análise fatorial de Componentes Principais (ACP)}

A análise das componentes principais procura descrever os dados contidos num quadro indivíduos-variáveis, isto é, a análise consiste em explicar a estrutura da variância e covariância de um conjunto de variáveis por meio de combinações lineares dessas mesmas variáveis. Assim, a ACP tem como propósito fornecer medidas responsáveis pelas maiores variações entre os resultados sem que se percam muita informação ao transformar o conjunto original de variáveis em outro (os componentes principais (CP) de dimensões equivalentes). Tal transformação ocorre com a menor perda de informação possível, sendo que esta também pode

\footnotetext{
${ }^{6}$ WARD J. Hierarchical grouping to optimize an objective function, Journal of the American Statistical Association. 1963;(58):236-244.
} 
auxiliar a eliminar algumas variáveis originais, no caso de possuírem pouca informação relevante. Essa abordagem possibilita a geração, seleção e interpretação das componentes investigadas, ainda auxilia a determinar as variáveis de maior influência na formação de cada componente. O grande número de informações sobre indivíduos deve ser transformado em um novo conjunto de variáveis que mantém, ao máximo, a variabilidade original do conjunto. Além disso, a ACP possibilita agrupar indivíduos similares mediante inspeções visuais em dispersões gráficas no espaço bi ou tridimensionais, o que facilita a interpretação geométrica. Esta redução da dimensionalidade é chamada transformação de Karhunnen-Loéve, ou análise de componentes principais, no qual os autovalores encontrados são denominados de componente principal.

O algoritmo se baseia na matriz de variância-covariância ou na matriz de correlação, de onde são extraídos os autovalores e os autovetores. Em suma, o que o método traduz, é a criação de novas variáveis não correlacionadas, obtidas de combinações lineares das variáveis iniciais que são apresentadas em ordem decrescente relativas ao seu poder de explicação. O processo parte da matriz de dados normalizados $\boldsymbol{X}_{n \times m}$, no qual sua dimensão é dada por $n$ casos explicados por $m$ variáveis. Assim, a matriz de correlação $(\boldsymbol{R})$ ou de variância-covariância amostral (S) são dadas por $\boldsymbol{S}_{m \times m}=\frac{1}{n-1}\left(\boldsymbol{X}_{m \times n}^{T} \boldsymbol{X}_{n \times m}\right)$ tal que

$$
\boldsymbol{S}=\left[\begin{array}{ccc}
S_{11} & \cdots & S_{1 j} \\
\vdots & \ddots & \vdots \\
S_{i 1} & \cdots & S_{i j}
\end{array}\right]
$$

Ainda, a matriz de correlação é representada por $\boldsymbol{R}_{m \times m}=\left[\begin{array}{ccc}1 & \cdots & r_{1 k} \\ \vdots & \ddots & \vdots \\ r_{k 1} & \cdots & 1\end{array}\right]$.

O cálculo do elemento dessa matriz pode ser obtido por meio da matriz $\boldsymbol{S}$, isto é:

$$
r_{i j}=\frac{s_{i j}}{\sqrt{s_{i i}} \sqrt{S_{j j}}}
$$

Algebricamente, segue-se para encontrar os autovalores $(\hat{\Lambda})$ - raízes características, bem como os autovetores $\left(\boldsymbol{W}_{m \times m}\right)$. Cada coluna de $\boldsymbol{W}$ é conhecida por componente principal, cuja ordem é determinada pelos respectivos autovalores. Nesse sentido, as primeiras colunas apresentam maior parcela da explicação da 
variância da amostra. Finalmente, a redução dos dados é possível com a criação da matriz das novas variáveis/escores $\left(\boldsymbol{T}_{n \times m}=\boldsymbol{X}_{n \times m} \boldsymbol{W}_{m \times m}\right)$. Contudo, apenas primeiras $r$ colunas de $\boldsymbol{W}$ determinam $W_{r}$ com $m$ linhas e $r$ colunas, tal que $r \leq m$. Então $\boldsymbol{X}_{(n \times m)} \boldsymbol{W}_{r(m \times r)}=\boldsymbol{T}_{r(n \times r)}$

Recomenda-se aplicar o teste Kaiser-Meyer-Olkin (KMO) para adequação dos dados. O teste compara a magnitude dos coeficientes de correlação parcial $\left(a_{i j}^{2}\right)$ do iésima variável com a j-ésimo fator com a correlação original i-ésima variável com o conjunto total. Então, o teste para adequação seque a expressão

$$
K M O_{j}=\frac{\sum_{i \neq j} r_{i j}^{2}}{\sum_{i \neq j} r_{i j}^{2}+\sum_{i \neq j} a_{i j}^{2}}
$$

Nesse sentido, seu valor entre 0 e 1, e valores superiores a 0,8 são sempre muito promissores. Juntamente com KMO, Reis (1997, p. 278) aponta outro teste que coaduna para checar a validade da aplicação deste tipo de análise. O Teste de esfericidade de Bartlett testa a hipótese de a matriz de correlações ser uma matriz identidade e seu determinante ser igual a 1. A hipótese nula do teste consiste em estabelecer que as variâncias são iguais para todas as variáveis. Então, para testar o grau de adequação dos dados ao método, consiste em verificar se a matriz $R$ é ou não uma matriz identidade. Espera-se que a hipótese nula - de presença de matriz identidade - seja rejeitada e, dessa forma, exista correlação entre as variáveis.

\subsubsection{Análise Fatorial}

Conforme Rummel (1967, p. 445) a análise fatorial "toma milhares e, potencialmente, milhões de medidas quantitativas e determina a ocorrência de padrões distintos entre elas" o que responde a questão qual padrão que explica a relação entre as variáveis. A análise fatorial procura por fatores capazes de explicar o que existe de comum nas variáveis originais. Para tanto, entende-se que o objeto principal da AF é identificar novas variáveis - fatores, em um número menor que o conjunto inicial, mas sem perda significativa de informações contidas no conjunto inicial das $m$ variáveis, além de classificar e reduzir observações. 
A AF permite tornar os dados observados mais claros, já que os mesmos são descritos por um grupo de categorias básicas, em número menor que as variáveis originais, chamados fatores. Os fatores são variáveis não observadas, escalas, itens, ou uma medida de qualquer espécie. Eles explicam a variância das variáveis originais que se revelam pelas correlações entre as variáveis que estão em análise. O método de extração destes fatores ${ }^{7}$, conforme Rumell (1967), é feito pela análise das componentes principais, descrita na sub-seção anterior.

Ambas, ACP e AF são técnicas de análise multivariada, aplicadas a um conjunto de variáveis, para descobrir quais dessas são mais relevantes na composição de cada fator, sendo estes independentes uns dos outros. Os fatores, que são gerados, serão utilizados de maneira representativa do processo em estudo no presente trabalho, e, para posteriores dados dos Censos a serem levantados no Rio Grande do Sul. Assim, Zambrano e Lima (2004, p. 556-7) salientam quatro etapas para realizar a técnica: a) normalização e verificação da adequação dos dados através da obtenção da matriz $R$, seguido dos testes KMO e Barlett; b) determinar o número de fatores necessários para representar o conjunto; c) rotação dos fatores e d) cálculo dos escores fatoriais.

A segunda etapa envolve a decisão do número de fatores necessários para representar o conjunto de dados. Como visto anteriormente, da matriz $\boldsymbol{R}$ são obtidos os autovalores e as proporções da variância total, utilizando-se o método de ACP. Dessa forma, os fatores são combinações lineares de variáveis, sendo o primeiro o que está associado à maior proporção da variância dos dados originais. Os componentes serão tantos quantos forem necessários até que se tenha explicado uma proporção razoável da variância. Geometricamente, os indicadores expressos em termos de vetores num sistema de coordenadas ortogonais agrupam-se em função de seu interrelacionamento. Admitindo-se que por esses agrupamentos de vetores passam eixos, os fatores na projeção dos vetores sobre os eixos, definem as cargas fatoriais dos indicadores sobre si. Conforme Hair (2005, p. 107), "uma carga fatorial representa uma correlação entre uma variável original e seu fator". Essas seguem uma interpretação semelhante a determinação da significância estatística de

\footnotetext{
${ }^{7}$ Para uma discussão mais ampla da metodologia ver Johnson e Wichern (1992).
} 
coeficientes de correlação. Assim, cada fator encontrado explica uma parcela da variância dos dados originais, e, como estão ordenados de forma decrescente, o primeiro fator explicará a maior proporção da variância dos dados.

Após identificar as variáveis que estão mais carregadas em um fator, os fatores precisam ser "batizados" em função das características reunidas. Cria-se uma matriz reduzida, a matriz de fatores, os quais explicam o que existe de comum entre as variáveis com a menor perda de informação possível. De certa forma, esta pode ser a fase mais sensível do trabalho, pois alguns fatores podem conter variáveis diversas com iguais comportamentos dificultando que se escolha adequadamente um nome que contemple o poder de explicação do fator. Ainda, determinam-se as comunalidades, isto é, a soma dos quadrados das cargas fatoriais dos indicadores sobre cada fator, exibindo a eficiência desses sobre a explicação da variabilidade total. Quanto mais próximo de 1 for o valor da comunalidade, maior parcela da variância original estará sendo captada pelo conjunto de fatores considerados. Resta ainda determinar o número de fatores a serem utilizados. Para o caso da utilização da matriz $\boldsymbol{R}$, tem que ser observado o critério da raiz latente ou autovalores, pois, segundo Zambrano e Lima (2004, p.559), "extraem-se apenas os fatores associados características maiores que 1", já os menores que a unidade são descartados.

Na terceira etapa, já selecionados os fatores e batizados, os mesmos são submetidos a uma rotação, mantendo a ortogonalidade entre eles. O objetivo da rotação ortogonal é o mesmo da geometria analítica, pois ela irá transformar os fatores obtidos em novos fatores independentes. A rotação altera a parcela de contribuição de cada fator, mas mantém os valores das comunalidades e a proporção de variância explicada pelo conjunto de fatores, pois, ela maximiza a carga de uma variável em um único fator e permite representar melhor cada fator.

A Rotação Varimax foi o método escolhido para rotacionar os eixos por ser um método bastante utilizado na literatura consultada. Ele produz um novo sistema de eixos ortogonais, conservando o número de fatores. Ele também permite que as variáveis reunidas apareçam com maior nitidez e facilitando a interpretação e análise. O método maximiza a soma das variâncias das cargas fatoriais e busca a simplificação das colunas da matriz fatorial. A simplificação máxima é conseguida se houver apenas 
cargas fatoriais próximas de 1 ou 0 . O coeficiente correlação, que varia entre +1 e -1 , indica a existência de associação positiva ou negativa entre fator e variável. Portanto, existe também a hipótese da ausência de associação entre ambos quando nenhuma relação linear for estabelecida.

Por fim, são calculados os escores fatoriais para cada observação. O escore fatorial ou índice é obtido pela multiplicação do valor padronizado da variável $n$ pelo coeficiente do escore fatorial correspondente. Estes valores constituem, exatamente, as projeções normalizadas dos pontos representativos das unidades sobre os eixos dos fatores que, por construção, tem média zero e desvio-padrão unitário. Dessa forma, Hair (2005) salienta que elevados escores fatoriais positivos ou negativos mostrarão que tal observação tem alta influência daquele fator.

\section{ANÁLISE DE RESULTADOS}

Nesta seção estão apresentados os resultados para a Análise de Agrupamento, Função Discriminante e Análise Fatorial proposta para os municípios que compunham os COREDEs agrícolas em 1995/96.

\subsection{Análise de Agrupamento (AA): os grupos iniciais}

Após estimativa das distâncias, foi preciso determinar o ponto em que o algoritmo aglomerativo deveria ser interrompido. Para isso, o ponto de corte que deu origem aos grupos formados ficou situado entre 179,37 e 275,06 - distância Euclidiana quadrada. Então, esse corte nos permitiu olhar a amostra dos 139 municípios pela ótica de apenas quatro grupos. Se comparados entre si, uma primeira característica é a de que dois deles apresentaram um número elevado de municípios, enquanto os demais são muito menores.

Os quatro grupos são compostos pelas respectivas cidades:

Grupo I: Alegrete, Itaqui, Rosário do Sul, Santana do Livramento, São Borja, São Gabriel, Uruguaiana e Cachoeira do Sul;

Grupo II: Giruá, Ajuricaba, Augusto Pestana, ljuí, Crissiumal, Três Passos, Sananduva, Tapejara, Cruz Alta, Ibirubá, Barros Cassal, Espumoso, Fontoura Xavier, Soledade; 
Grupo III: Panambi, Não-Me-Toque; Quaraí, Cerro Branco, Paraíso do Sul, Restinga Seca, São Sepé, Vila Nova do Sul, Bossoroca, Caibaté, Cerro Largo, Entre-ljuís, Eugênio de Castro, Garruchos, Guarani das Missões, Porto Xavier, Salvador das Missões, Santo Ângelo, Santo Antônio das Missões, São Luiz Gonzaga, São Miguel das Missões, Bom Progresso, Campo Novo, Catuípe, Chiapeta, Condor, Coronel Barros, Coronel Bicaco, Inhacorá, Jóia, Pejuçara, Santo Augusto, São Martinho, Tenente Portela, Tiradentes do Sul, Boa Vista das Missões, Dois Irmãos das Missões, Engenho Velho, Erval Seco, Frederico Westphalen, Gramado dos Loureiros, Seberi, Água Santa, Ibiaça, Ibiraiaras, Lagoa Vermelha, Colorado, Fortaleza dos Valos, Lagoa dos Três Cantos, Quinze de Novembro, Saldanha Marinho, Salto do Jacuí, Santa Bárbara do Sul, Selbach, Tapera, Alto Alegre, Nicolau Vergueiro, Mormaço e Victor Graeff, Roque Gonzales, Alpestre, São José do Ouro; e

Grupo IV: São Pedro do Butiá, Irai, Itacurubi, Manoel Viana, Dezesseis de Novembro, Pirapó, São Nicolau, Vitória das Missões, Barra do Guarita, Braga, Derrubadas, Humaitá, Miraguaí, Redentora, São Valério do Sul, Sede Nova, Vista Gaúcha, Ametista do Sul, Caiçara, Cerro Grande, Jaboticaba, Lajeado do Bugre, Liberato Salzano, Nonoai, Novo Tiradentes, Palmitinho, Pinhal, Pinheirinho do Vale, Planalto, Rio dos Índios, Rodeio Bonito, Sagrada Família, Taquaruçu do Sul, Três Palmeiras, Trindade do Sul, Vicente Dutra, Vista Alegre, André da Rocha, Barracão, Cacique Doble, Caseiros, Esmeralda, Machadinho, Maximilhano de Almeida, Paim Filho, Santo Expedito do Sul, São João da Urtiga, Tupanci do Sul, Campos Borges, Gramado Xavier, Ibirapuitã, Itapuca, Lagoão e São José do Herval.

Sendo assim, têm-se quatro grupos que perfazem os 139 municípios da amostra selecionada. Encerramos a primeira etapa do agrupamento com uma a amostra dividida em diferentes grupos, com um primeiro, o menor da amostra, contendo oito municípios apenas. O grupo II é um pouco maior (15), mas os grupos III (61) e IV (55) apresentam ainda mais municípios. A chance de termos incluído algum município por engano nos grupos maiores é grande. Então, o estudo passa para a etapa de verificar a função discriminante dos grupos. 


\subsection{Função Discriminante: checks and balances}

Como um refinamento da técnica de Análise de Agrupamento, a Função discriminante, revelou que alguns municípios, em primeira instância, foram mal classificados pela AA. Dessa forma, determinados municípios estavam em posição de migração intragrupos estando localizados nos limites das divisões entre os grupos pelas três funções geradas.

O novo retrato revelou que o grupo I manteve-se sólido, ratificando a homogeneidade entre os municípios do grupo, e a heterogeneidade em relação aos demais grupos. O grupo II foi ampliado com a entrada de dois novos municípios. Panambi e Não-Me-Toque deixaram o grupo III e, dessa forma, completaram o estágio de transição para uma agricultura menos tradicional.

O grupo III ao perder dois elementos para o grupo II, recebeu três novos municípios oriundos do grupo IV: São Pedro do Butiá, Irai e Mormaço. O grupo IV conta com três novos municípios que vieram do grupo III: Roque Gonzáles, Alpestre e São José do Ouro.

A metodologia refinou os resultados gerados pela Análise de Agrupamentos e, dessa forma, através das funções geradas, delimitou com maior precisão a área de abrangência entre cada grupo ${ }^{8}$ no mapa territorial em que os municípios foram agrupados.

\subsection{Análise Fatorial (AF) e Componentes Principais (CP)}

Cabe lembrar, que fazem parte da amostra 139 municípios do estado do Rio Grade do Sul sob a ótica de 17 variáveis - já discriminadas no Quadro 01 - sendo esses municípios pertencentes aos COREDEs essencialmente agrícolas ${ }^{9}$, conforme classificação da Fundação de Economia e Estatística (FEE) junto ao Governo do Estado do Rio Grande do Sul - Rumos 2015 (2005). Dessa forma seguem-se os passos descritos por Zambrano e Lima (2004).

\footnotetext{
${ }^{8}$ No ANEXO B estão sumariamente dispostos os grupos e seus respectivos componentes.

${ }^{9}$ Segundo Rumos 2015 (2005) esses são: a) Fronteira Oeste, com 10 municípios; b) Jacuí Centro, com 6 municípios; c) Missões, 21 municípios; d) Noroeste Colonial, 29 municípios; e) Médio Alto Uruguai, também, com 29 municípios, f) Noroeste, 18 municípios, g) Alto Jacuí e h) Alto da Serra do Botucaraí com, 12 e 14 municípios, respectivamente.
} 
O primeiro passo buscou verificar a adequação dos dados ao método de análise fatorial através dos testes KMO e de Esfericidade. O KMO revelou um valor de 0,816, indicando adequação suficiente dos dados ao método. Por sua vez, o teste de Barlett of Sphericity $(4097,40)$ mostrou-se significativo a $1 \%(p<0,01)$, assim, rejeitando-se a hipótese nula - $\left(H_{0}: P=1\right)$. Desse modo, ambos os testes realizados permitem concluir que a amostra realizada é adequada ao procedimento da análise fatorial.

Prosseguindo na análise dos Componentes Principais (CP) a serem encontrados através da matriz $R$, com no máximo de 17 componentes possíveis. Temos os principais componentes em relação às principais variáveis relacionadas, a saber:

a) CP 1: PEA $(0,713) ; \operatorname{ASSPEC}(0,589) ; \operatorname{ASSAGR}(0,492) ; \operatorname{TRATOR}(0 ; 934) ; \operatorname{ADUBO}$ $(0,523) ; \quad$ IRRIGA $(0,768) ; \operatorname{RECVEG}(0,904) ; \operatorname{RECANI}(0,879) ; \operatorname{FINANC}(0,660)$; MAQCOL $(0,867) ; \operatorname{VALVEG}(0,922) ; \operatorname{VALANI}(0,903) ; \operatorname{AREXPL}(0,866)$ e GADO $(0,827)$;

b) CP 2: POP (- 0,590); $\operatorname{ASSPEC~(-0,546);~} \operatorname{ASSAGR~}(-0,732) ; \operatorname{ADUBO}(-0,717)$;

c) CP 3: (RENDA; 0,724);

d) CP 4: (DISTRG; 0,805); POP $(0,573)$.

Para todos os CP, as correlações foram significativas com um nível de significância de 95\%. Dessa forma, a análise não mostrou mais CPs relevantes, sendo o descarte de mais de uma dezena apropriado. Ainda, para delimitar com exatidão o número apropriado, observamos os mesmos pelos valores das raízes características autovalores. Assim, a escolha do número de fatores que iremos utilizar será dado pelos autovalores maiores que 1 .

A segunda etapa envolve então a decisão do número de fatores necessários para representar o conjunto de dados. Para isso, segue-se o critério da raiz latente ou autovalores, pois extraem-se apenas os fatores a associados às raízes características maiores que 1. Assim, garantimos o descarte dos fatores com autovalores menores que a unidade.n A Tabela 1, a fim de complementar as informações do estudo, 
apresenta as raízes características, bem como a parcela de variância explicada pelos fatores - não rotacionados.

Tabela 1 - Raízes características - autovalores - da matriz de correlação simples (17 x 17) para os determinados COREDEs - 1995/96

\begin{tabular}{lcccc}
\hline Fatores & Raiz Característica & $\begin{array}{c}\text { \% Total da } \\
\text { Variância }\end{array}$ & $\begin{array}{c}\text { Raiz Característica } \\
\text { Acumulada }\end{array}$ & $\begin{array}{c}\text { \% da Variância } \\
\text { Acumulada }\end{array}$ \\
\hline 1 & 9,044 & 53,200 & 9,044 & 53,200 \\
2 & 2,562 & 15,068 & 11,606 & 68,268 \\
3 & 1,469 & 8,639 & 13,074 & 76,907 \\
4 & 1,098 & 6,458 & 14,172 & 83,365 \\
\hline
\end{tabular}

Fonte: Resultados da pesquisa obtidos através do Statistica.

Assim, os quatro primeiros autovalores, os que melhor representam o conjunto, explicam aproximadamente $83,37 \%$ da variância do conjunto. Dessa forma, optou-se por quatro fatores explicativos para as variáveis originas. O primeiro fator, que é a combinação linear das variáveis originais, explicou individualmente a maior parcela da variância do conjunto. O fator 1 captou aproximadamente 53,20\% da variância, enquanto o segundo fator explica pouco mais de 15\% da variância.

Como salientando, o método Varimax objetiva melhorar a interpretação dos fatores. Realizando a rotação nos quatro fatores escolhidos têm-se:

Tabela 2 - Percentagem da variância explicada por cada fator após a rotação ortogonal.

\begin{tabular}{lcc}
\hline Fatores & Antes da rotação (\%) & Rotacionado (\%) \\
\hline 1 & 53,200 & 38,523 \\
2 & 15,068 & 21,875 \\
3 & 8,639 & 15,043 \\
4 & 6,458 & 7,925 \\
Total & 83,365 & 83,365 \\
\hline \multicolumn{2}{l}{ Fonte: Resultados da pesquisa obtidos através do Statistica. }
\end{tabular}

Efetuada a rotação, fica evidente que o fator 1 passou a captar uma proporção menor da variância total das variáveis originais. Entretanto, o poder explicativo do fator 2 e 3 melhoraram significativamente, da mesma forma que o fator 4 teve seu poder de explicação elevado, mas em menor intensidade que os fatores 2 e 3 . A rotação equilibrou o poder de explicação, sem alterar o total da variância, que permanece igual ao valor anterior à rotação.

$\mathrm{Na}$ Tabela 3 estão apresentadas as cargas fatoriais ou os coeficientes de correlação entre cada fator e cada uma das 17 variáveis. Também, está a proporção 
de variância das variáveis originais explicadas por cada um dos quatro fatores, após a rotação pelo método Varimax.

Tabela 3 - Cargas fatoriais, comunalidades e percentagem da variância explicada por cada fator após a rotação.

\begin{tabular}{|c|c|c|c|c|c|}
\hline \multirow{2}{*}{$\begin{array}{l}\text { Indicadores de } \\
\text { Modernização }\end{array}$} & \multicolumn{4}{|c|}{ Cargas fatoriais/Coeficiente de Correlação } & \multirow{2}{*}{ Comunalidade } \\
\hline & Fator 1 & Fator 2 & Fator 3 & Fator 4 & \\
\hline POP & $-0,022$ & 0,568 & $-0,003$ & 0,573 & 0,608 \\
\hline PEA & 0,508 & 0,801 & $-0,090$ & $-0,052$ & 0,952 \\
\hline ASSPEC & 0,186 & 0,755 & 0,292 & 0,005 & 0,665 \\
\hline ASSAGR & 0,001 & 0,799 & 0,215 & 0,374 & 0,753 \\
\hline TRATOR & 0,628 & 0,461 & 0,540 & 0,161 & 0,984 \\
\hline ADUBO & 0,179 & 0,928 & $-0,039$ & $-0,060$ & 0,951 \\
\hline IRRIGA & 0,757 & 0,150 & 0,145 & 0,255 & 0,860 \\
\hline RECVEG & 0,736 & 0,239 & 0,519 & 0,091 & 0,994 \\
\hline RECANI & 0,903 & 0,247 & 0,131 & 0,002 & 0,942 \\
\hline FINANC & 0,520 & $-0,077$ & 0,672 & 0,145 & 0,701 \\
\hline MAQCOL & 0,506 & 0,439 & 0,654 & 0,139 & 0,978 \\
\hline VALVEG & 0,741 & 0,297 & 0,493 & 0,084 & 0,994 \\
\hline VALANI & 0,945 & 0,189 & 0,140 & 0,073 & 0,993 \\
\hline AREXPL & 0,963 & 0,077 & 0,133 & 0,074 & 0,992 \\
\hline RENDA & 0,011 & 0,040 & 0,807 & $-0,059$ & 0,471 \\
\hline DISTRG & $-0,248$ & 0,004 & $-0,042$ & $-0,842$ & 0,463 \\
\hline GADO & 0,970 & 0,042 & 0,060 & 0,061 & 0,991 \\
\hline $\begin{array}{l}\text { \% da variância } \\
\text { após a rotação }\end{array}$ & 38,523 & 21,875 & 15,043 & 7,926 & \\
\hline
\end{tabular}

Os valores absolutos poderiam ser adotados conforme Hair (2005, p.107), ou seja, superiores a 0,50, em virtude do número a amostra de 139 municípios. Contudo, como ocorre que mais de um fator tem correlação significativa com determinada variável - como é o caso da PEA nos fatores 1 e 2 - optou-se por considerar apenas aqueles com valores superiores a 0,60. Dessa forma, as cargas fatoriais fornecem informações para a caracterização dos fatores comuns.

Assim temos que o fator 1 associa-se forte e positivamente com o número de tratores (TRATOR; 0,628), valor das receitas advindas de produtos vegetais e animais (RECVEG; 0,736 e RECANI; 0,903), valor da produção vegetal e animal (VALVEG; 0,741 e VALANI; 0,945), área explorada nos municípios (AREXPL; 0,963), efetivo bovino (GADO; 0,970) e, por fim, a variável que mede irrigação (IRRIGA; 0,757). Portanto, o fator pode ser associado a uma medida da modernização da agricultura, de forte relação com os 
insumos modernos e caráter capital-intensivo. Dessa forma, o fator 1 é aquele que serve como uma medida de modernização da agropecuária associada ao uso intensivo da terra em municípios da fronteira. Possuem em comum um elevado rebanho bovino conjugado com lavouras de arroz, além de dimensões territoriais acentuadas se comparada aos demais municípios.

Observa-se que 0 fator 2 está correlacionado com a população economicamente ativa (PEA; 0,801), contudo mais fortemente ligada a esse fator, assistência técnica especializada na produção animal (ASSPEC; 0,755), assistência técnica especializada na produção vegetal (ASSAGR; 0,799) e número de estabelecimentos que utilizam adubos químicos (ADUBO; 0,928). Em suma, o fator 2 pode ser interpretado como o fator que mede a modernização da agropecuária associado ao apoio para a inovação tecnológica e químico-biológica, além de denotar um caráter trabalho-intensivo. O fator 2 pode ser visto como o fator "apoiado pela inovação". Dessa forma, os fatores supracitados apresentam uma semelhança. Ambos estão medindo o processo de modernização da agropecuária na região estudada por dois ângulos. O primeiro fator, capta mais esse processo em termos de resultados dependentes da produção predominante, enquanto o segundo fator capta o fenômeno mais na dimensão do apoio à inovação e assistência na agricultura e pecuária.

O fator 3 apresenta correlação positiva e forte com o valor dos financiamentos (FINANC; 0,672), número de máquinas para colheita (MAQCOL; 0,654) e com o índice de renda dos municípios (RENDA; 0,807). Assim, RENDA e FINANC caracterizam o fator 3 , e este é batizado como o fator que mede o processo modernizante pela ótica financeira, ou seja, "nível de renda e valores financiados dos municípios".

Por fim, o fator 4, único com associações negativas, tem correlação forte e negativamente com a variável distância do porto de Rio Grande (DISTRG; - 0,842). Ele ainda tem uma relação com a variável POP $(0,573)$ e muito fraca com a área explorada $(0,074)$. As evidências apontam para relação espacial que esses municípios mantêm em relação à distância do porto de Rio Grande, por onde escoa a maior parte da produção agropecuária exportada gaúcha. Entende-se que quanto maior a distância para o escoamento da produção agropecuária voltada ao mercado externo, menor o 
nível de modernização. Sendo assim, esse fator assume a função "espacial" que é um componente fundamental na competitividade. Ele também mostra que esses municípios tem grandes populações e pouca área explorada pela agricultura de insumos modernos.

Como o objetivo da técnica de análise multivariada é uma redução de dados para facilitar a interpretação/conclusões acerca de fenômenos, em especial, a análise fatorial e de agrupamentos, busca-se simplificar a matriz de dados $\boldsymbol{X}_{139 \times 17}$. De fato, a análise de cluster se deteve em reduzir o número de linhas enquanto a análise fatorial reduziu o número de variáveis - colunas. Foram criadas 4 novas variáveis - os fatores - que juntas explicam aproximadamente $83,37 \%$ da variância do conjunto. Assim sendo, como os 139 municípios foram agrupados em 4 grupos, o mais homogêneo possível, é correto inferir que os grupos formados caracterizam em média os municípios neles contidos. Sendo assim, antiga matriz com 139 linhas/municípios e 17 colunas/variáveis da amostra foi reduzida a 4 grupos e 4 novas variáveis - os fatores. Tem-se a nova matriz gerada após a aplicação da técnica multivariada, e, consequente e pretendida redução do número inicial de dados. Isto só foi possível pela complementação de uma técnica a outra, ou seja, agrupando-se primeiro para logo explicar as razões deste agrupamento pelos fatores gerados na análise fatorial. Assim, a matriz inicial de dados foi reduzida aos dados dispostos na Tabela 4.

Tabela 4 - Classificação em ordem decrescente do nível tecnológico, participação percentual no valor da produção agropecuária, animal e vegetal para os quatro grupos homogêneos dos COREDEs agrícolas do Estado do Rio Grande do Sul.

\begin{tabular}{lccccccccc}
\hline Grupo & Fator 1 & Fator 2 & Fator 3 & Fator 4 & $\begin{array}{c}\text { Número de } \\
\text { Municípios }\end{array}$ & $\begin{array}{c}\text { \% VAB* } \\
\text { agropecuária }\end{array}$ & $\begin{array}{c}\% \text { VBP } \\
\text { animal }\end{array}$ & $\begin{array}{c}\% \text { VBP } \\
\text { vegetal }\end{array}$ \\
\hline I & 3,614 & 0,068 & 0,538 & 0,180 & 8 & $5,76 \%$ & 9,36 & 14,55 & 11,85 \\
II & $-0,343$ & 2,049 & 0,322 & 0,413 & 16 & $11,50 \%$ & 6,96 & 6,20 & 7,52 \\
III & $-0,220$ & $-0,214$ & 0,409 & 0,142 & 60 & $43,17 \%$ & 13,96 & 12,31 & 14,60 \\
IV & $-0,186$ & $-0,373$ & $-0,619$ & $-0,301$ & 55 & $39,57 \%$ & 6,52 & 5,42 & 5,78 \\
\hline \multicolumn{7}{c}{ N } & & \multicolumn{7}{c}{139} & $100 \%$ & & & \\
\hline
\end{tabular}

Fonte: Resultados da pesquisa obtidos através do Statistica.

* Valor adicionado bruto agropecuário dos municípios em 1995, dado fornecido pela Fundação de Economia e Estatística - FEE.

Depreende-se que os municípios que formam o grupo I (escore de 3,614) apresentam uma moderna e dinâmica agricultura voltada para o comercial - indústria e exportação - e exploração da pecuária conjuntamente pelo fato de terem o maior 
escore no fator 1. Esse escore coaduna com a realidade dos municípios do grupo, pois são zonas tradicionais de produção de produtos do agronegócio gaúcho. Os grupos II, III e IV, por sua vez, estão em um patamar diferente do grupo I ao não exibirem escore positivo com o fator receita e pecuária de corte.

Os resultados pela análise multivariada são verossímeis, pois o grupo I formado de apenas oito municípios é responsável por 9,36\% do VAB do Rio Grande do Sul, ainda, representava 14,55\% e $11,85 \%$ do valor bruto da produção animal de grande porte e vegetal do RS. Freitas, Paz e Nicola (2007) chegaram a um resultado proporcionalmente semelhante, ou seja, um pequeno grupo representa uma parcela importante do valor de produção.

O grupo II, por sua vez, reponde por 6,96\% do VAB do Estado e 6,20\% e 7,52\% da produção animal e vegetal respectivamente. O grupo III tem $13,96 \%$ do VAB do RS, contudo, é bom lembrar que a representatividade bem superior aos demais, deve ao fato de este grupo conter aproximadamente $43,17 \%$ da amostra selecionada para 0 estudo. O grupo IV, apesar de tamanho elevado, 39,57\% da amostra, responde por $6,52 \%$ do VAB do estado, o que corrobora com a sua relação com os fatores 1 e 2 , ou seja, tem uma relação inversa a modernização e, também, com o apoio a inovação.

Já o grupo II, pelo seu escore no fator $1(-0,343)$ denota não ser intensivo em insumos como irrigação e uso da terra para produção animal de corte. Contudo, o fator 2 (2,049) revela uma agricultura também dinâmica tendo um apoio a modernização tecnológica e químico-biológica, vide sua relação com as variáveis que mensuram os estabelecimentos com assistência técnica e utilização de adubos.

Já os grupos III e IV, não possuem uma estrita ligação com caráter de agropecuária moderna. Ainda, os grupos III e IV, demonstram ser os grupos que contêm a produção menos dinâmica, se comparado aos grupos I e II. Depreende-se que os elementos do grupo III, apesar do baixo escore para o fator $2(-0,214)$ e com o fator $1(-0,220)$, se comparado ao fator 4 . Eles demonstram um padrão de transição para uma agricultura mais dinâmica apoiada na modernização, e estão no intermediário entre os grupos II e IV.

Quanto ao escores para os grupos referentes ao fator 3 - nível de renda e financiamentos - pode inferir-se que: 
a) o grupo I coadunando com a elevada representatividade econômica, apresenta o mais elevado nível de renda e valores em financiamentos $(0,538)$;

b) o grupo II para esse fator está mais abaixo em relação ao grupo III (0,322);

c) o grupo III apresenta valores mais elevados que o grupo II $(0,409)$, reforçando a ideia de transição, pois se verifica uma renda mais elevada e valores de financiamentos superiores que o já estabelecido grupo Il;

d) o grupo IV se encontra em situação longe de grandes cultivos intensivos em solo ou crédito. Por se tratar de COREDE agrícola, esse não tem elevados valores em financiamentos, número de implementos e assistência, o que reforça o caráter mais tradicional da produção nesses municípios.

Por fim, o grupo Il é o grupo com melhor relação com o fator espacial, pois tem o escore mais elevado $(0,413)$. O grupo I obteve escore de $(0,180)$, não tão alto quanto o grupo II, pois, neste grupo estão os municípios que em grande parte estão localizados na fronteira do estado com Argentina e Uruguai - fronteiras tradicionais de produção gaúcha. O grupo III obteve escore de $(0,142)$ seguido do grupo IV $(-0,301)$, que obteve pior escore dos quatro grupos, esse então sendo formados pelos municípios com maior distância do porto de Rio Grande. Assim, considerando que a distância do porto de Rio Grande é um fator de competitividade, levando em conta que $25 \%$ da produção se perde, antes mesmo, de chegar ao embarque no porto (MELO, 1990). Os municípios do grupo II apresentaram assim uma vantagem competitiva em relação aos outros.

Finalizada a análise ${ }^{10}$, considerando as informações quantitativas dos municípios e suas realidades/peculiaridades, há elementos suficientes para apontar uma concentração da agricultura moderna e da agropecuária no grupo I. Uma situação semelhante para o grupo II, excluindo as variáveis ligadas a bovinocultura. $\mathrm{O}$ grupo III agrega potencial de transição da agricultura mais tradicional para uma com apoiada na inovação. A análise dos dados do Censo revelou também um grupo com o menor grau de modernização da agricultura do estado. Baixa exploração da área, e,

\footnotetext{
${ }^{10}$ No ANEXO 3 pode ser encontrado o resultado de estudo na forma de uma mancha de modernização.
} 
em virtude de seu elevado tamanho, o grupo carece de uma atenção especial para explorar possíveis potencialidades entre seus municípios. Partindo de Lopes e Rocha (2005), podemos associar aos municípios do grupo IV um padrão de agricultura mais tradicional, o que explicaria parte do comportamento refletido à época.

\section{CONCLUSÕES}

Os resultados obtidos apontam uma série de evidências sobre a realidade da modernização brasileira no período em análise. Conforme a revisão de literatura, os resultados apontam que o padrão de modernização, excludente e conservador, também se repetiu no estado. Uma minoria de municípios, oito, dispõem de elevado grau de modernização na agricultura, bem como são responsáveis por aproximadamente $14,55 \%$ do valor bruto da produção Inimal e $11,85 \%$ do valor bruto de produção vegetal. Essa realidade ratifica a elevada concentração da agropecuária no grupo I.

Por outro lado, há municípios da amostra que guardam pouca relação com a pecuária e produção arroz, os quais estão concentrados fora do grupo I. O grupo II apresentou um tamanho médio, com 16 municípios, e mostrou relação forte com a agricultura dinâmica e apoiada no uso de adubos, assistência técnica e modernização tecnológica. O grupo III, apesar de seu elevado tamanho, está em um estágio que pode ser entendido de transição entre a agropecuária mais tradicional, para uma apoiada na modernização e em insumos mais modernos, dessa forma, tomando um caráter mais dinâmico da agropecuária. O resultado mais agudo diz respeito ao grupo IV, que contém 55 municípios, apresentando a menor relação com os dois fatores que medem a modernização da agropecuária pela ótica de insumos modernos e apoio a modernização - fatores 1 e 2 -, bem como pela ótica financeira.

Quanto ao nível de renda e financiamentos, novamente o grupo I se destaca, pois seus oito municípios concentram 37\% do valor dos financiamentos. O grupo III tem resultado maiores que o grupo II, reiterando a sua condição de transição a uma agricultura mais dinâmica e apoiada em tecnologia na agricultura. Novamente, o grupo IV é o de pior resultado para financiamentos e renda. 
O fator espacial, que buscou interagir entre a distância do porto e da cidade produtora, se mostrou eficiente. De modo que, se a competitividade está relacionada a menores custos de transporte também, o grupo II se mostrou o mais competitivo nesse aspecto.

Com base no estudo, infere-se que os municípios do grupo I são os que têm melhor nível de modernização da agricultura e pecuária, demonstrando, em virtude de suas longas histórias de fronteiras agrícolas gaúcha, consolidados no cenário do Rio Grande do Sul. O grupo II encontra-se num padrão dinâmico de agrícola, seguido do grupo III que, no período estudado, demonstra uma transição entre agricultura tradicional e dinâmica.

Por fim, observa-se que o grupo IV tem o menor grau de modernização da agropecuária do estado e sem exploração intensiva da área tanto para agricultura como para pecuária. Em virtude de seu elevado tamanho, carece de uma atenção especial para que eventuais potencialidades voltadas para a agricultura tradicional possam ser exploradas.

Para trabalhos futuros, após a publicação do Censo Agropecuário 2007/08 e 2017, sugere-se um novo retrato da modernização agrícola no Rio Grande do Sul. Com isso, será possível comparar se persiste a concentração da modernização nas áreas do grupo I e, se, os municípios do grupo III conseguiram ingressar em um grupo mais dinâmico. Ainda, salienta-se que a inclusão ou exclusão de perguntas nos questionários do censo podem alterar o número de variáveis para o estudo. Por exemplo, o Censo de 2017 tem informação mais detalhada sobre irrigação, acrescentando o tipo de mecanismo, como o número de pivôs e bombas. Com isso, os grupos I e II poderiam ser melhor estudados, isto é, seria possível reforçar as conclusões de que os municípios do grupo I estão relacionados com a produção de arroz e bovinocultura de corte, enquanto o grupo II teria relação com a produção de soja e outras culturas. 


\section{REFERÊNCIAS}

BANDEIRA P. Participação, Articulação de Atores Sociais e Desenvolvimento Regional. Brasília: Texto para discussão IPEA.1999;(630). Available from: http://www.ipea.gov.br/portal/images/stories/PDFs/TDs/td_0630.pdf.

FREITAS CA, PAZ MV, NICOLA DS. Avaliando a intensidade da modernização da agropecuária gaúcha: uma aplicação de análise fatorial e cluster. Revista Análise Econômica. 2007;25(47):121-149.

FUNDAÇÃO DE ECONOMIA E ESTATÍSTICA - FEE. [Internet]. Porto Alegre. Idese. Available from em: <http://www.fee.tche.br>.

FÜRSTENAU V. O Crédito Rural no Brasil e seus efeitos sobre a agricultura gaúcha (1965-84). Porto Alegre: Fundação de Economia e Estatística Sigfried Emanuel Hauser, 1988:109 p.

GAMA H. O renascimento do transporte hidroviário no RS. Voto. 2007; 3(35): p.26-29.

GRAZIANO DSJ. A nova Dinâmica da Agricultura Brasileira. Campinas: UNICAMP; 1996.

GUIA 4 RODAS. Guia 4 Rotas: Distâncias em km [Internet]. Available from: http://www. guia4rodas.abril.com.br/mapasbrasi. 2007.

HAIR JF. Análise multivariada de dados. 5th ed. Porto Alegre: Bookman; 2005.

HÄRDLE W, SIMAR L. Applied Multivariate Statistical Analysis. [Internet]. Berlin and Louvain-la-Neuve; 2003. Available from: <http://www.xplore-stat.de>.

INSTITUTO BRASILEIRO DE GEOGRAFIA E ESTATÍ́STICA - IBGE. [Internet]. Rio de Janeiro. Censo Agropecuário. Available from: <http://www. ibge.gov.br>.

JOHNSON RA, WICHERN DW. Applied Multivariate Statistical Analysis. 3 rd ed. New Jersey: Prentice Hall; 1992.

LOPES IV, ROCHA DP. Agricultura familiar: muitos produzem pouco. In: Conjuntura Econômica. 2005;59(02):30-35.

MASSUQUETTI A. A Mudança no padrão de financiamento da agricultura brasileira [dissertation]. Porto Alegre: Economia Rural/UFRGS; 1998. 222p.

MELO FBH. O crescimento agrícola brasileiro dos anos 80 e as perspectivas para os anos 90. Revista de Economia Política. 1990;10(3):22-30.

MINGOTI SA. Análise de dados através de métodos de estatística multivariada: uma abordagem aplicada. Belo Horizonte: Editora UFMG; 2005. 
MORRISON DF. Multivariate statistical methods. 2 nd ed. New York: Mc Graw Hill; 1976.

MÜLLER CAA. História Econômica o Rio Grande do Sul. Porto Alegre: Ed. Grande Sul; 1999.

RANGEL I. Questão agrária, industrialização e crise urbana no Brasil. Porto Alegre: Editora da UFRGS; 2004.

REIS E. Estatística Multivariada. Lisboa: Edições Silabo Ltda; 1997.

RIO GRANDE DO SUL. Rumos 2015: estudo sobre desenvolvimento regional e Logística de Transportes no RS. [CD-ROM]; 2005 mai. 97 p.

RUMMEL RJ. Undestanding factor analysis. Journal of Conflict Resolution, 1967; 11(4):444-480.

SCHNEIDER S, FIALHO AAV. Pobreza rural, desequilíbrios regionais e desenvolvimento agrário no Rio Grande do Sul. Teoria e Evidência Econômica. Passo Fundo, v.8, n.15, p. 117-150, nov. 2000.

ZAMBRANO C, LIMA JE. Análise Estatística Multivariada de Dados Socioeconômicos. In: SANTOS ML, VIEIRA WC editors. Métodos quantitativos em economia. Viçosa: Editora UFV; 2004. 


\section{ANEXO A - COREDEs utilizados para o estudo}

Os municípios fazem parte dos COREDEs essencialmente agrícolas, conforme classificação da Fundação de Economia e Estatística (FEE) junto ao Governo do Estado do Rio Grande do Sul (2005) são: a) Fronteira Oeste, com 10 municípios; b) Jacuí Centro, com 6 municípios; c) Missões, 21 municípios; d) Noroeste Colonial, com 29 municípios; e) Médio Alto Uruguai, também, com 29 municípios, f) Nordeste, com 18 municípios, g) Alto da Serra do Botucaraí, com 14 municípios e, h) Alto Jacuí, com 12 municípios. O que pode ser verificado na ilustração.

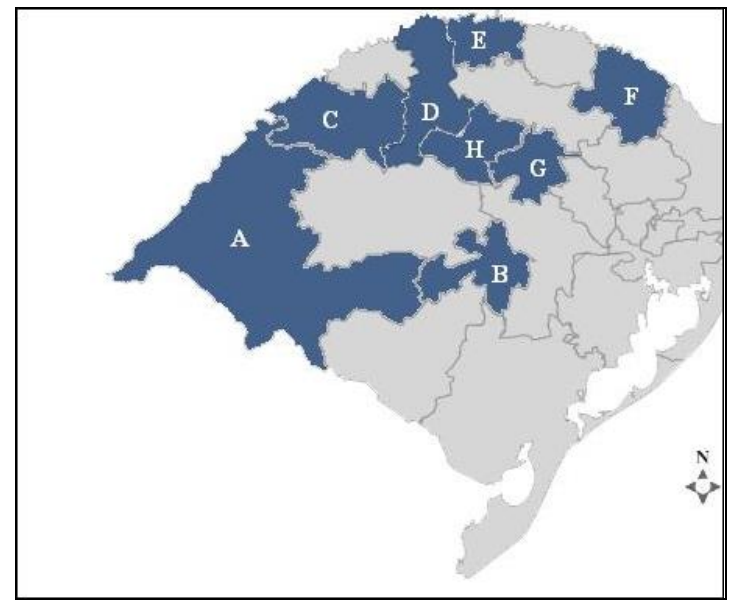

Fonte: FEE

\section{ANEXO B - Grupos formados}

i) Grupo I: Alegrete, Itaqui, Rosário do Sul, Santana do Livramento, São Borja, São Gabriel, Uruguaiana e Cachoeira do Sul;

ii) Grupo II: Giruá, Ajuricaba, Augusto Pestana, ljuí, Crissiumal, Três Passos, Sananduva, Tapejara, Cruz Alta, Ibirubá, Barros Cassal, Espumoso, Fontoura Xavier, Soledade; Panambi, Não-Me-Toque;

iii) Grupo III: Quaraí, Cerro Branco, Paraíso do Sul, Restinga Seca, São Sepé, Vila Nova do Sul, Bossoroca, Caibaté, Cerro Largo, Entre-ljuís, Eugênio de Castro, Garruchos, Guarani das Missões, Porto Xavier, Salvador das Missões, Santo Ângelo, Santo Antônio das Missões, São Luiz Gonzaga, São Miguel das Missões, Bom Progresso, Campo Novo, Catuípe, Chiapeta, Condor, Coronel Barros, Coronel Bicaco, Inhacorá, Jóia, Pejuçara, Santo Augusto, São Martinho, Tenente Portela, Tiradentes do Sul, Boa Vista das Missões, Dois Irmãos das Missões, Engenho Velho, Erval Seco, Frederico Westphalen, Gramado dos Loureiros, Seberi, Água Santa, Ibiaça, Ibiraiaras, 
Lagoa Vermelha, Colorado, Fortaleza dos Valos, Lagoa dos Três Cantos, Quinze de Novembro, Saldanha Marinho, Salto do Jacuí, Santa Bárbara do Sul, Selbach, Tapera, Alto Alegre, Nicolau Vergueiro, São Pedro do Butiá, Irai, Mormaço e Victor Graeff; e

iv) Grupo IV: Itacurubi, Manoel Viana, Roque Gonzales, Alpestre, Dezesseis de Novembro, Pirapó, São Nicolau, Vitória das Missões, Barra do Guarita, Braga, Derrubadas, Humaitá, Miraguaí, Redentora, São Valério do Sul, Sede Nova, Vista Gaúcha, Ametista do Sul, Caiçara, Cerro Grande, Jaboticaba, Lajeado do Bugre, Liberato Salzano, Nonoai, Novo Tiradentes, Palmitinho, Pinhal, Pinheirinho do Vale, Planalto, Rio dos Índios, Rodeio Bonito, Sagrada Família, Taquaruçu do Sul, Três Palmeiras, Trindade do Sul, Vicente Dutra, Vista Alegre, André da Rocha, Barracão, Cacique Doble, Caseiros, Esmeralda, Machadinho, Maximilhano de Almeida, Paim Filho, Santo Expedito do Sul, São João da Urtiga, Tupanci do Sul, Campos Borges, Gramado Xavier, Ibirapuitã, Itapuca, Lagoão, São José do Ouro e São José do Herval.

\section{ANEXO C - Mancha de Modernização dos Municípios que pertencem aos COREDEs essencialmente agrícolas}

Os municípios fazem parte dos COREDEs essencialmente agrícolas, conforme classificação da Fundação de Economia e Estatística (FEE) junto ao Governo do Estado do Rio Grande do Sul (2005) segundo divisão política de 1993/94. Pois, ao período posterior diversos distritos se emanciparam e, dessa forma, os dados do novo Censo terão um acréscimo de municípios dos referidos COREDEs.

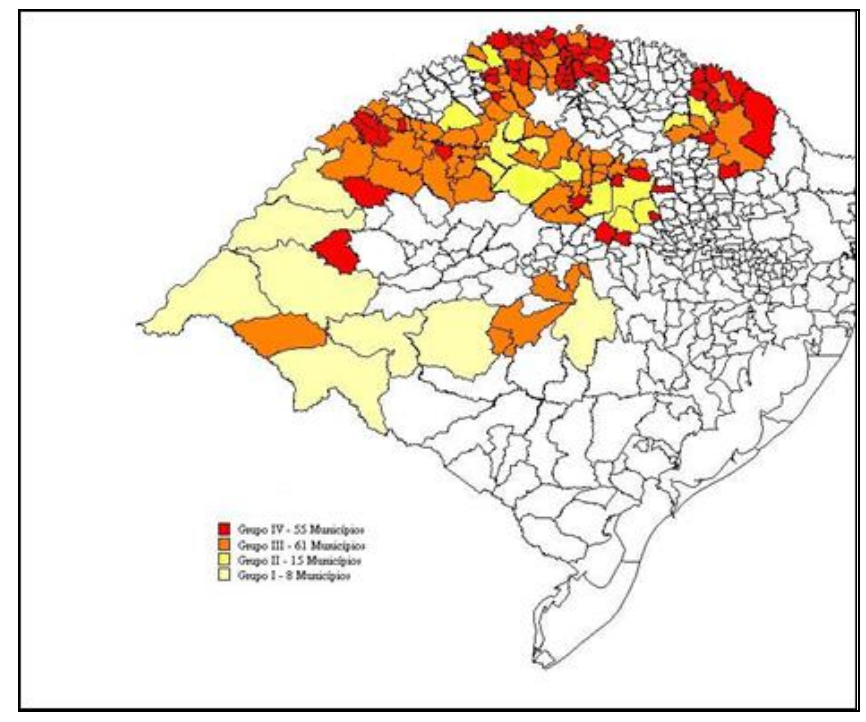

Fonte: Elaboração própria 